\title{
Valor do exame citológico per-operatório em neurocirurgias
}

Primeira submissão em 14/11/03 Última submissão em 11/02/05 Aceito para publicação em 28/03/05 Publicado em 20/06/05

\section{The value of per-operative cytological exam in neurosurgeries}

Ana Helena Pereira Correia'; José Carlos Pando Esperança²; Marcello Reis da Silva3; Jorge Marcondes de Souza ${ }^{4}$; Leila Chimelli ${ }^{5}$

\begin{abstract}
unitermos Neurocirurgia

Esfregaço

Diagnóstico per-operatório

\section{resumo}

Introdução: Mais de $90 \%$ dos resultados citológicos per-operatórios neurocirúrgicos correlacionam-se com o histopatológico. Porém, a sua real necessidade é por vezes questionada. Objetivo: Avaliar a necessidade do exame per-operatório tendo-se em vista a conduta neurocirúrgica imediata. Material e métodos: Revimos 130 casos de exames per-operatórios solicitados de um total de 293 procedimentos cirúrgicos para tratamento de lesões expansivas do sistema nervoso central (SNC), extra ou intra-axiais, sendo 46 biópsias estereotáxicas e 84 cirurgias abertas, realizados de 1998 a 2001. Foram realizados esfregaços, o diagnóstico foi comparado com o histopatológico, e os neurocirurgiões, questionados sobre a implicação do resultado do exame na conduta, caso a caso. Resultados: O exame per-operatório foi solicitado em $44,4 \%$ do total de cirurgias, tendo sido considerado relevante para a conduta em $84 \%$ destes casos ou, nas biópsias, para avaliar a representatividade do material, o que corresponde a $37,2 \%$ do total de cirurgias realizadas no período. Excluindo-se as biópsias estereotáxicas, com indicação inquestionável, o exame foi necessário em $75 \%$, sendo considerado desnecessário, sobretudo nas lesões extra-axiais, em geral solicitado por curiosidade do cirurgião. Conclusão: Apesar de ser um exame com acurácia demonstrada, a realização de exames per-operatórios sem utilidade para a conduta neurocirúrgica pode vir a sobrecarregar patologistas em tarefas desnecessárias. Nossos resultados demonstraram que o exame foi necessário na grande maioria dos casos, mesmo após a exclusão das biópsias estereotáxicas, sobretudo nas lesões intra-axiais, auxiliando na avaliação da demanda desses exames, por vezes excessiva, apesar de útil para treinamento e ensino.
\end{abstract}

Background: More than $90 \%$ of neurosurgical per-operative cytological results have a good correlation with histopathology. However, its real necessity is sometimes questioned. Objective: Evaluate the necessity of per-operatory exam regarding immediate neurosurgical procedure. Material and methods: We reviewed 130 per-operative exams requested out of 293 surgical procedures for expansive extra or intra-axial CNS lesions, 46 stereotactic biopsies and 84 open surgeries, performed from 1998 to 2001. Smears were prepared, the results compared to the histopathological data and then the neurosurgeons were argued about its necessity and influence upon the surgical procedure, case by case. Results: The per-operative exam was requested in $44.4 \%$ of the total number of surgeries performed, $84 \%$ of which were relevant for the procedure, or, in biopsies, to evaluate sample representativeness, corresponding to $37.2 \%$ of the total number of surgeries performed in that period. Once stereotactic biopsies were excluded, considering that in such cases the per-operative exam is unquestionable, the previous value dropped to 75\%; the per-operative smear was considered unnecessary mainly in extra-axial lesions, usually required because of neurosurgeons' curiosity. Conclusion: Despite its well-known accuracy, per-operative exams performed without a real necessity for the surgical procedure could overcharge pathologists unnecessarily. Our results have shown that the exam was useful in the great majority of cases, even excluding stereotactic biopsies; they also help us evaluate the demand for per-operative exams, sometimes excessive, although important for practicing and teaching purposes.

\section{key words} Neurosurgery Smear Per-operative diagnosis

\footnotetext{
1. Médica do Serviço de Anatomia Patológica do Hospital Universitário Clementino Fraga Filho (HUCFF).

2. Professor-assistente do Departamento de Patologia da Faculdade de Medicina da Universidade Federal do Rio de Janeiro (UFRI), HUCFF.

3. Médico do Serviço de Neurocirurgia do HUCFF.

4. Professor-adjunto do Departamento de Cirurgia da UFR); chefe do Serviço de Neurocirurgia do HUCFF.

5. Professora-titular do Departamento de Patologia da Faculdade de Medicina da UFRJ/ HUCFF.

Estudo realizado no Serviço de Anatomia Patológica do HUCFF da UFR).
} 


\section{Introdução}

O exame per-operatório microscópico, realizado pela técnica do esfregaço, é uma ferramenta bastante útil para o neurocirurgião, pois é um método rápido e que ajuda no melhor planejamento da cirurgia. O exame microscópico per-operatório, seja pela técnica do esfregaço ou pela realização de cortes congelados, é amplamente utilizado desde a sua introdução no início do século $20^{(9,24,37)}$. As vantagens do exame citológico (esfregaço) sobre o corte congelado incluem a maior rapidez (em geral é realizado em cerca de 5 minutos), o menor custo, pois não necessita de aparelhagem para corte nem de técnico especializado, e, em se tratando de suspeita de lesões infecciosas, evita a contaminação do criostato( ${ }^{(15,27,31)}$. No caso específico das neurocirurgias, onde as amostras são em geral escassas e diminutas, o exame citológico apresenta mais vantagens em relação ao corte congelado, pois requer menor quantidade de material, permitindo a preservação de tecido para inclusão em parafina e para a realização de estudo imuno-histoquímico. Além disso, a consistência amolecida do tecido do sistema nervoso central (SNC) torna fácil a realização de esfregaços ${ }^{(33)}$. O exame citológico também permite a análise de todo o material selecionado, diferentemente da congelação, em que apenas um pequeno segmento do material selecionado é examinado, pois em geral apenas um ou dois cortes consecutivos são realiza$\operatorname{dos}^{(7)}$, o que pode resultar em problemas no diagnóstico per-operatório, já que as neoplasias do SNC podem apresentar aspectos variegados ${ }^{(20)}$, dependendo da região analisada. $\mathrm{O}$ esfregaço fornece ainda maior preservação de detalhes nucleares e citoplasmáticos, que são perdidos durante a congelação, e esses detalhes são fundamentais para a exclusão de processos não-neoplásicos do SNC ou, no caso das neoplasias, para a determinação do tipo celular de origem ${ }^{(8,11)}$, cujas características no esfregaço já foram amplamente descritas $(2,4,5,10,13,21,25,29,34,36)$.

Apresenta, ainda, elevada acurácia, já que, quando realizados por patologistas experientes, vários estudos demonstraram haver mais de $90 \%$ de correlação positiva do esfregaço com o resultado final do material incluído em parafina $a^{(3,7,11,14,27,28,32,35,36)}$, sendo essa também a nossa realidade. No caso das biópsias estereotáxicas, indicadas em casos de lesões da linha média inacessíveis à remoção cirúrgica direta ou lesões que tendem a infiltrar estruturas vitais adjacentes ${ }^{(22)}$, o exame per-operatório é imprescindível para confirmar se o posicionamento da agulha orientado por estereotaxia é adequado, ou seja, se o material obtido é representativo de lesão ${ }^{(18,19)}$. Entretanto, várias alterações reativas do sistema nervoso ou, ainda, estruturas encefálicas normais podem mimetizar neoplasias - é o caso da camada granular interna do cerebelo, em que pese o diagnóstico diferencial com linfomas, e a gliose reacional, que pode ser confundida com gliomas. Porém, exceto nos casos de biópsias estereotáxicas, a necessidade do exame per-operatório é por vezes questionada, por sobrecarregar patologistas em tarefas desnecessárias ou que não interferem nas condutas imediatas por parte dos neurocirurgiões.

\section{Objetivo}

O presente trabalho tem por objetivo avaliar a real necessidade do exame citológico per-operatório em neurocirurgias, nos casos em que a presença do patologista foi solicitada para este fim, correlacionando o resultado de tal exame com a conduta neurocirúrgica.

\section{Materiais e métodos}

Foi realizado estudo retrospectivo de 130 exames peroperatórios de lesões intracranianas e raquianas, extra e intra-axiais, de pacientes de ambos os sexos, com idades variando entre 3 e 77 anos, de um total de 293 procedimentos cirúrgicos para tratamento de lesões expansivas do SNC, no período de 1998 a 2001. Em 45 dos 130 casos, o procedimento foi biópsia estereotáxica, e nos 85 casos restantes, cirurgia aberta.

Em todos os casos, foram realizados esfregaços. Para a confecção dos esfregaços, o material obtido durante a neurocirurgia era examinado macroscopicamente, e um ou mais fragmentos eram selecionados para exame per-operatório, sendo o restante do material e as amostras adicionais fixados em formol a $10 \%$ tamponado, para processamento de rotina para cortes histológicos. Os fragmentos selecionados para o esfregaço, medindo cerca de $1 \mathrm{~mm}^{3}$, eram colocados próximo à extremidade de uma lâmina e, com outra lâmina colocada paralelamente sobre a primeira, realizava-se 0 esfregaço através de compressão e deslizamento. Para cada caso foram realizadas duas a quatro lâminas, imediatamente fixadas em álcool absoluto durante 1 minuto. Após rápida lavagem em água corrente, as lâminas eram coradas com hematoxilina durante 1 minuto e novamente lavadas em água corrente. Em seguida, era realizada lavagem em álcool absoluto, e as lâminas, coradas com eosina durante 50 segundos. Procediam-se, então, a cinco lavagens em álcool absoluto, mais cinco rápidas em xilol e finalmente as lâminas eram montadas com bálsamo e lamínula. 
Alternativamente, as lâminas eram coradas com azul de toluidina, procedendo-se de forma semelhante até a fixação em álcool absoluto e posterior lavagem em água corrente. Depois desta etapa, as lâminas eram coradas com azul de toluidina a $1 \%$ durante 1 minuto, depois lavadas em água corrente, passando rapidamente em álcool a 70\%, álcool absoluto (duas vezes), xilol (duas vezes) e finalmente montadas com bálsamo e lamínula, conforme descrito previamente por Chimelli e Campos $(1993)^{(7)}$.

De posse das requisições dos exames e dos resultados, foi questionada e discutida com os neurocirurgiões a necessidade ou não de cada exame, sob o ponto de vista do impacto que o resultado do esfregaço teria sobre a conduta neurocirúrgica imediata. Quanto à necessidade do exame e aos fatores que o justificassem, foram feitas as seguintes perguntas sobre cada caso: 1. No caso em questão, o exame per-operatório foi necessário para a conduta neurocirúrgica imediata? 2. Em caso afirmativo, justifique (ex.: se serviu para definir natureza da lesão, grau histológico de neoplasia, limite com o tecido são, avaliar representatividade do material, etc.). As respostas foram obtidas de comum acordo entre os neurocirurgiões.

\section{Resultados}

\section{Correlação entre exame per-operatório e con- duta neurocirúrgica imediata}

O exame per-operatório foi solicitado em 130 das 293 cirurgias realizadas no período, ou seja, em $44,4 \%$ dos casos (Figura 1). Em 109 dos 130 casos estudados (84\% dos casos de exame per-operatório, o que corresponde a $37,2 \%$ do total de procedimentos cirúrgicos realizados), o exame per-operatório foi importante para a definição de condutas ou, no caso das biópsias estereotáxicas, para avaliar a representatividade do material, ou seja, se o material obtido era suficiente para conclusão diagnóstica, se não no esfregaço, pelo menos após a inclusão em parafina (Figura 2). Excluindo-se os 46 casos de biópsias estereotáxicas, cuja indicação do esfregaço não é questionada, o exame citológico per-operatório foi importante para a conduta em 63 casos (75\% dos casos de exame per-operatório, o que corresponde a $28,7 \%$ do total de procedimentos cirúrgicos do período) (Figura 3).

Correlacionando-se os esfregaços per-operatórios com o corte histológico correspondente, observou-se que nos meningiomas, por exemplo, o arranjo concêntrico de algumas células, por vezes com pseudo-inclusão nuclear, limites citoplasmáticos pouco nítidos e até mesmo a presença de psamomas, facilitou o diagnóstico citológico. Nos esfregaços per-operatórios de tumores astrocíticos, o fundo fibrilar dos prolongamentos citoplasmáticos, a celularidade, as atipias nucleares e o aspecto dos vasos, sobretudo a proliferação endotelial, foram valorizados na graduação das lesões. No glioblastoma, além desses critérios, havia também áreas de necrose. Já nos esfregaços exibindo gliose reacional, a presença de astrócitos com prolongamentos citoplasmáticos muito nítidos, de aspecto estrelado, sem os demais comemorativos descritos acima para os tumores astrocíticos, permitiu o diagnóstico.

\section{Análise qualitativa dos exames}

Fora as biópsias estereotáxicas, o exame foi considerado necessário, sobretudo em lesões intra-axiais (Tabela 1), para definir a natureza da lesão, se primária ou metastática, e para graduar histologicamente tumores primários (lesões de alto e baixo graus). No caso dos microadenomas

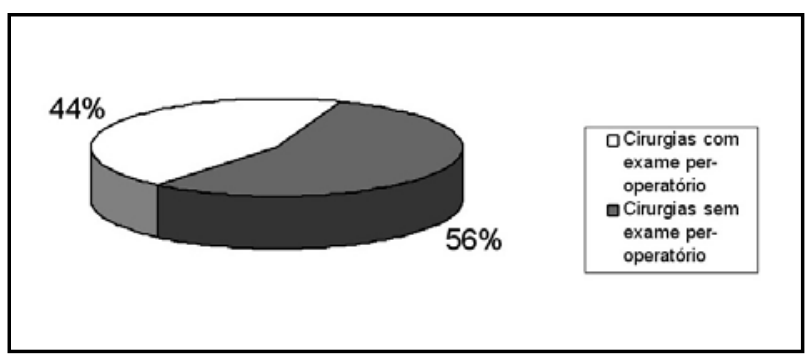

Figura 1 - Percentual de procedimentos neurocirúrgicos (lesões expansivas) com exame per-operatório (total de 293 cirurgias)

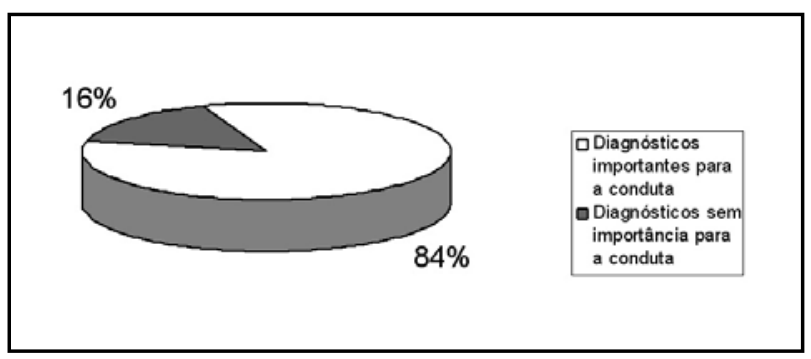

Figura 2 - Importância do diagnóstico per-operatório para a conduta neurocirúrgica imediata (total de 130 casos)

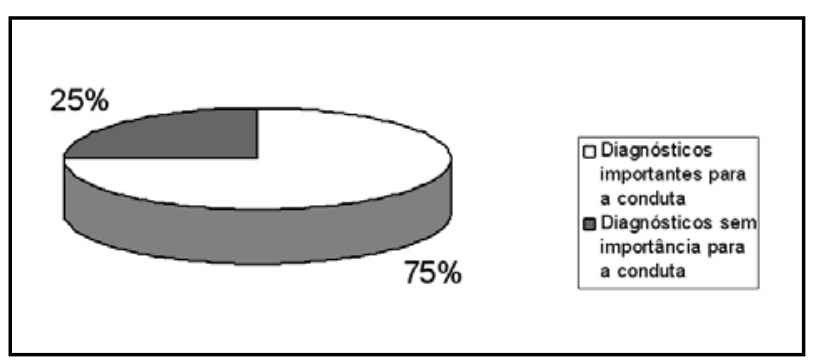

Figura 3 - Importância do diagnóstico per-operatório para a conduta neurocirúrgica imediata excluindo biópsias estereotáxicas (total de 63 casos) 
de hipófise, o esfregaço serviu para orientar se o material examinado era o adenoma ou a adeno-hipófise normal. Em 21 dos 130 casos estudados (16\%), o exame foi considerado desnecessário (Figura 2). Tais casos correspondiam principalmente a lesões extra-axiais (meningiomas, schwannomas), conforme demonstrado na Tabela 2.

\section{Discussão}

Quando realizado por patologistas experientes, o esfregaço mostra alto percentual de concordância com o diagnóstico final do material incluído em parafina. No presente trabalho, este percentual de correlação positiva esfregaço/histopatológico foi de mais de $90 \%$, permanecendo, portanto, entre a faixa de $83 \%$ a $94 \%$ descrita por outros autores ${ }^{(8,11,22)}$.

Apesar de ser um exame com acurácia demonstrada, tem-se questionado a sua real necessidade, ou seja, até que ponto seria capaz de afetar a conduta neurocirúrgica imediata. Nossos resultados demonstraram que o exame foi necessário, isto é, afetaria a conduta neurocirúrgica, na grande maioria dos casos, ou seja, 84\%. Embora tenha diminuído após a exclusão dos casos de biópsia estereotáxica - na qual o exame é fundamental para avaliar a representatividade do material -, este percentual manteve-se alto, 75\%. No grupo de casos com importância para o diagnóstico, sobressaem-se as lesões intra-axiais, em que pese a diferenciação entre tumor primário do SNC e metástase, e entre neoplasias do SNC de alto e baixo graus. Isto porque, no caso de lesões metastáticas únicas, a ressecção completa das mesmas é recomendada sempre que possível, pois afeta favoravelmente a sobrevida ${ }^{(6)}$, o mesmo acontecendo com tumores primários de baixo grau, em que o prognóstico encontra-se intimamente relacionado à capacidade de ressecção dos mesmos ${ }^{(17,26)}$. Nos casos de reoperação, 0 exame é necessário, pois as características anatômicas e macroscópicas não permitem boa diferenciação entre a neoplasia e o tecido nervoso adjacente. Com relação a lesões de etiologia infecciosa, o exame foi importante para avaliar representatividade do material, identificar $o$ agente (p. ex., criptococose) e definir a conduta, já que a drenagem deve ser a mais ampla possível para facilitar a penetração de agentes antimicrobianos e no tratamento posterior ${ }^{(1,12,30)}$. Nos casos de linfoma, o tratamento principal não é cirúrgico, mas sim a associação de radioterapia e quimioterapia ${ }^{(16)}$, sendo as ressecções em geral mais restritas, visando apenas à obtenção de material suficiente para diagnóstico adequado, o que muitas vezes requer coloração para reticulina e estudos imuno-histoquímicos. A importância do diagnóstico per-operatório desta neoplasia reside em evitar ressecções desnecessárias e, ao mesmo tempo, avaliar se a amostragem é suficiente para exames posteriores adicionais à rotina de hematoxilina e eosina (HE). No presente trabalho, em dois casos de linfoma (total de três), foi realizado estudo complementar pela reticulina, e em um deles procedeu-se a estudo imuno-histoquímico.

Os poucos casos de lesões extra-axiais em que o exame per-operatório foi considerado importante incluíram três meningiomas e um schwannoma. Em dois casos de meningiomas, o exame foi considerado necessário para

Tabela 1 Exames per-operatórios com importância para a conduta cirúrgica imediata

Esfregaços per-operatórios: total de 130 casos

Com importância para a conduta cirúrgica imediata: 109

46 biópsias estereotáxicas, 63 cirurgias abertas, das quais:

- 9 glioblastomas

- 7 astrocitomas pilocíticos

- 5 ependimomas

- 5 carcinomas metastáticos

- 4 adenomas hipofisários

- 3 oligodendrogliomas

- 3 linfomas

- 3 meningiomas

- 2 astrocitomas anaplásicos

- 2 carcinomas do trato sinunasal

- 2 oligodendrogliomas anaplásicos
Outros - um de cada

Xantoastrocitoma pleomórfico, tuberculoma

DNT, astrocitoma de baixo grau

Neurocisticercose, angiolipoma

Subependimoma, adeno-hipófise normal

Córtex cerebelar normal e fibrose meníngea

Schwannoma, ganglioglioma anaplásico

Neurocitoma central, germinoma

Osteoblastoma agressivo, aspergilose

Criptococose meníngea, pineoblastoma

Paquimeningite hipertrófica idiopática 
Exames per-operatórios sem importância para a conduta cirúrgica

Tabela 2 imediata

Esfregaços per-operatórios: total 130 casos

Sem importância para a conduta cirúrgica imediata: 21

- 9 meningiomas

- 4 schwannomas

- 4 adenomas hipofisários

- 1 hemangioblastoma

- 1 hemangioma cavernoso

- 1 ependimoma

- 1 processo inflamatório granulomatoso

o diagnóstico diferencial de lesão única com carcinoma metastático; no terceiro caso, o exame foi importante para diagnóstico diferencial com carcinomatose meníngea, pois se tratavam de múltiplas lesões e, caso este diagnóstico fosse confirmado, seria implantado cateter para administração de agente quimioterápico no mesmo tempo cirúrgico. No caso do schwannoma, tratava-se de lesão expansiva aderida à raiz nervosa de $L 4$, com erosão do corpo vertebral, sendo necessário afastar meningioma, caso em que a raiz nervosa poderia ser poupada durante a ressecção, já que nas lesões intraneurais isto não é possível.

Os casos de exame per-operatório desnecessário incluíam, sobretudo, lesões extra-axiais (meningiomas, schwannomas), conforme demonstrado na Tabela 2, visto que, salvo raras exceções, não implicaria condutas diferentes. Em alguns casos, apesar de lesões intra-axiais, o exame foi considerado desnecessário (Tabela 2). Em um hemangioblastoma cerebelar e num hemangioma cavernoso da região da pineal, ambos com aspecto, ao exame de imagem, bastante característico, a ressecção completa estava indicada. Uma recidiva de ependimoma do ângulo pontocerebelar configurava lesão com diagnóstico prévio. Em um caso de processo inflamatório granulomatoso selar/supra-selar com diagnóstico diferencial com neoplasia hipofisária, a ressecção completa estaria indicada nas duas situações, seja para facilitar a penetração de agentes antimicrobianos, seja para tratamento da neoplasia.

\section{Conclusão}

O diagnóstico per-operatório influenciou a conduta cirúrgica na maioria dos casos. Nos casos de biópsia estereotáxica, mesmo quando o diagnóstico não puder ser conclusivo durante o ato operatório, o patologista pode informar se a biópsia representa tecido patológico ou não. Além disso, os resultados obtidos auxiliam também o posicionamento dos patologistas diante da demanda, por vezes excessiva, de tal exame, apesar de útil para treinamento e ensino. O patologista com experiência em neuropatologia cirúrgica, atuando em conjunto com a equipe de neurocirurgia e avaliando as informações clínicas e os exames de imagem, é capaz de influenciar as decisões e condutas a serem tomadas.

\section{Referências}

I. BERNARDINI, G. L. Diagnosis and management of brain abscess and subdural empyema. Curr Neurol Neurosci Rep, v. 4, n. 6, p. 448-56, 2004.

2. BLEGGI-TORRES, L. F. et al. Pleomorphic xantoastrocytoma: report of a case diagnosed by intraoperative cytopathological examination. Diagn Cytopathol, v. 24, n. 2, p. I20-2, 200 I.

3. BLEGGI-TORRES, L. F. et al. Accuracy of the smear technique in the cytological diagnosis of 650 lesions of the central nervous system. Diagn. Cytopathol, v. 24, n. 4, p. 293-5, 2001

4. BLEGGI-TORRES, L. F. et al. Dysembrioplastic neuroepithelial tumor: cytological diagnosis by intraoperative smear preparation. Diagn. Cytopathol, v. 26, n. 2, p. 92-4, 2002.

5. CAMPOS, I. S.; CHIMELLI, L. Aspecto citológico de tumores intracranianos e do canal vertebral. Arq Neuropsiquiatr, v. 5I, n. 2, p. 196-204, 1993.

6. CARTER, B. S.; HARSH, G. R. Diagnosis of suspected intracranial metastases. Role of direct tissue examination. Neurosurg Clin NAm, v. 7, n. 3, p. 425-33, 1996.

7. CHIMELLI, L.; CAMPOS, I. S. Valor do esfregaço no diagnóstico per-operatório dos tumores provenientes de neurocirurgias. Arq Neuropsiquiatr, v. 5I, n. 2, p. 190-5, 1993.

8. COLLAÇO, L. M. et al. Stereotactic biopsy and cytological diagnosis of solid and cystic intracranial lesions. Cytopathology, v. |4, n. 3, p. 131-5, 2003.

9. DUDGEON, L. S.; PATRICK, C.V. A new method for the rapid microscopical diagnosis of tumors: with an account of 200 cases so examined. Br J Surg, v. I5, p. 250, 1927.

10. DVORACEK, M. A., KIRBY, P. A. Intraoperative diagnosis of tanycytic ependymoma: pitfalls and differential diagnosis. Diagn Cytopathol, v. 24, n. 4, p. 289-92, 200 I.

I I.FOLKERTH, R.D.Smears and frozen sections in the intraoperative diagnosis of Central Nervous System lesions. Neuropathology, 
v. 5, n. I, p. I-18, 1994

12. FUENTES, S. et al. Management of brain stem abscess. $\mathrm{Br} J$ Neurosurg, v. 15, n. I, p. 57-62, 2001.

13. GOH, S. G.; CHUAH, K. L. Role of intraoperative smear cytology in the diagnosis of anaplastic oligodendroglioma. A case report. Acta Cytol, v. 47, n. 2, p. 293-8, 2003.

14. GUAJARDO, R. G.; QUINTANA, O. B.; RODRÍGUEZ, J. A. Citología por impronta en el diagnóstico transoperatorio de los tumores del sistema nervioso central. Patología, v. 33, p. 163-6, 1995.

15. GUARDA, L. A. Intraoperative cytologic diagnosis: evaluation of 370 consecutive intraoperative cytologies. Diagn Cytopathol, v. 6, p. 235-42, 1990.

16. HOCHBERG, F.H. et al.The therapy of primary brain lymphoma. J Neurooncol, v. 10, p. 191, 1991.

17. KELLY, P.J.; HUNT, C. The limited value of cytoreductive surgery in elderly patients with malignant gliomas. Neurosurgery, v. 34, n. I, p. 62-6, 1994.

18. KEPES,J.J. Pitfalls and problems in the histopathologic evaluation of stereotactic needle biopsy specimens. Neuropathology, v. 5, p. 19-33, 1994.

19. KITCHEN, N. D.; BRADFORD, R. MCLAUGHIN, J. E.The value of per-operative smear examination during sterotactic biopsy. Acta Neurochirurgica, v. 121, p. 196-8, 1993.

20. KLEIHUES, P.; CAVENEE, W.K. Pathology and genetics: tumours of the central nervous system (WHO). France: IARC Press, 2000.

2I. KRISTT, D. A. Pathologic evaluation of fresh tissue from neurosurgical biopsies: technical and interpretational aspects. Isr J Med Sci, v. 32, p. I252-5, 1996.

22. LIWNICZ B. H.; RODRIGUEZ C. A. The central nervous system aspiration biopsy: cytologic interpretation and histologic bases. In: KOSS, L. G;WOYKE, S.; OLSZEWSKI,W. (eds.) NY: Igaka-Shoin Medical Publishers, 1984. p. 457-90.

23. LIWNICZ, B. H. et al. Needle aspiration cytology of intracranial lesions: a review of 84 cases. Acta Cytol, v. 26, p. 779, 1982.

24. MACCARTHY,W. C.The diagnostic reliability of frozen sections. Am J Pathol, v. 5, p. 377, 1929.

25. NAMIKI, T. S. et al. Stereotaxic biopsy diagnosis of central nervous system lymphoma. Am J Clin Pathol, v. 90, n. I, p. 40-5, 1988.

26. NAZZARO, J. M.; NEUWELT, E. A. The role of surgery in the management of supratentorial intermediate and high-grade astrocytomas in adults. J Neurosurg, v. 73, n. 3, p. 331-44, 1990.

27. OLASODE, B. J.; IRONSIDE, J.W. The brain smear, a rapid affordable intraoperative diagnostic technique for brain tumours appropriate for Africa. Trop Doct, v. 34, n. 4, p. 223-5, 2004.

28. ROESSLER, K. et al. High diagnostic accuracy of cytologic smears of central nervous system tumors. A I5-year experience based on 4172 patients. Acta Cytol, v. 46, n. 4, p. 667-74, 2002.

29. SALINERO, E.; BELTRAND, L.; COSTA, J. R. Intraoperative cytologic diagnosis of chordoid meningioma. A case report. Acta Cytol, v. 48, n. 2, p. 259-63, 2004.

30. SCHWARTZ, S.;THIEL, E. Update on the treatment of cerebral aspergillosis. Ann Hematol, v. 83, n. I, p. 42-4, 2004.

31. SCOPA, C. D. et al. Tissue imprints in surgical pathology: a rapid intraoperative diagnostic aid. Diagn Cytopathol, v. 6, p. 5-8, 1990.

32. SILVERMAN, J. F. et al. Cytologic results of fine-needle aspiration biopsies of the central nervous system. Cancer, v. 58, n. 5 , p. III7-21, 1986.

33. SLOWINSKI, J.; HARABIN-SLOWINSKA, M.; MROWKA, $\mathrm{R}$. Smear technique in the intra-operative brain tumor diagnosis: its advantagens and limitations. Neurol Res, v. 2I, n. I, p. $121-4,1999$.

34. SUGITA, Y. et al. Cytodiagnosis of central neurocytoma in intraoperative preparations. Acta Cytol, v. 48, n. 2, p. 194-8, 2004.

35.TARATUTO,A. L.; SEVLEVER, G.; PICCARDO, P. Clues and pitfalls in stereotactic biopsy of the central nervous system. Arch Pathol Lab Med, v. I I5, n. 6, p. 596-602, 1991.

36. TORRES, L. F. B.; COLLAÇO L. M. Diagnóstico citopatológico de tumores neuroepiteliais pela técnica do esfregaço. Arq Neuropsiquiatr, v. 59, p. 173-9, 1992.

37. WILSON, L. B. A method of rapid preparation of fresh tissues for the microscope. JAMA, v. 45, p. 1737, 1905. 